\title{
Modeling and Adapting Production Environmental Stress Testing
}

\author{
Toby Joyce, Oonagh Gaffney, and Dr. Shirish Kher, Alcatel-Lucent \\ Simon Wilson, Trinity College, Dublin
}

\begin{abstract}
This study describes the production sampling environmental stress test (PSEST) process and the offline analysis conducted. Some of the key characteristics and parameters of the test are outlined. The analytical process is based on two types of regression model, each of which links a dependent variable (the log of time to failure in each dwell, or the log of the number failed in each dwell) to independent variables such as temperature and age. These two types of regression are known as Weibull regression ${ }^{1}$ and Poisson regression. ${ }^{2}$ Using the estimated regression coefficients, expressions for the probability distribution of a unit under test can be written and used to optimize the test duration.
\end{abstract}

\section{KEYWORDS}

Environmental stress testing, temperature cycling, Weibull regression, generalized linear models

\section{BACKGROUND}

Environmental stress tests (ESTs) are commonly applied during the design and manufacture of telecommunications and electronic equipment to screen for design and manufacturing defects. In these processes, environmental stresses are applied to the units under test. Design EST (DEST) usually occurs during the product development process, with the objective of correcting design flaws, thereby "ruggedizing" the hardware. Production sampling EST (PSEST) is typically used as an in-line manufacturing check, often on a sampling basis, to precipitate and detect latent process-related defects, thereby eliminating so-called infant mortality failures. PSEST also provides early information on field performance and design improvement opportunities. DEST and PSEST procedures are conducted on optical-, copper-, and wireless-based network products.

PSEST is the second part of a two-stage activity, with DEST as a prerequisite. Highly accelerated life testing (HALT) is a major part of DEST. ${ }^{3}$ The production analogue of HALT is highly accelerated stress screening (HASS). While HALT has become a common activity in the design phase of most electronic products, HASS is still rarely employed in manufacturing testing. Although product manufacture is commonly outsourced to large contractors, these contractors may not have sufficient capacity to perform HASS in medium- to high-volume production on a continuous basis. Because of the lack of economies of scale, it is difficult to justify employing HASS unless the product is of very small dimensions or low volume.

Companies that already use traditional chambers with mechanical refrigeration may be unwilling to make the large investment needed to set up HASS chambers, which require liquid nitrogen cooling for rapid transitions and have high running costs. On the other hand, HALT activities during design can be scheduled and performed conveniently by a contractor with the required specialty equipment. PSEST can be performed within the parameters of the older style of testing. While this results in a lesser level of stress applied to production packs, if accompanied by effective analysis PSEST can be just as successful as HASS in the long term. 


\section{PSEST PROGRAMS}

The products covered in this study are the circuit packs installed in customized cabinets to make up a network infrastructure. Circuit packs consist of at least one printed wiring board with mounted electronic components attached by means of surface-mount or plated through-hole technologies. Packs may range in size from a few square inches to 2 square feet, and in weight from a few grams to a few kilograms. Packs may be installed in backplanes or boxes for home, curbside, remote countryside, or central office use in customer applications.

PSEST is carried out after initial testing and before final testing. Failed units are repaired and retested from the start of the test. Upon completing the full duration of the test failure-free, circuit packs are shipped to customers. In early product life there may be emphasis on defect screening, but information on product performance is also used to improve design.

Circuit packs are assembled and tested in the factory or by subcontractors. Not all packs undergo PSEST. The circuit packs are classified according to the level of temperature-cycling stress applied during PSEST:

- High. Packs installed outdoors, and thus exposed to temperatures ranging from as low as $-40^{\circ} \mathrm{C}\left(-40^{\circ} \mathrm{F}\right)$ to as high as $60^{\circ} \mathrm{C}\left(140^{\circ} \mathrm{F}\right)$.

- Medium. Packs installed in central office environments, and thus subjected to temperature cycles between $-5^{\circ} \mathrm{C}\left(23^{\circ} \mathrm{F}\right)$ and $50^{\circ} \mathrm{C}\left(122^{\circ} \mathrm{F}\right)$.

- Low. Some central office packs receive only an elevated-temperature or room-ambient burn-in. This study will not address these pack types in detail.

Random vibration does not form part of PSEST testing, but it does form an important part of the DEST suite of tests.

Packs are mounted in environmental chambers with the temperature controlled by mechanical cooling and heating equipment. Packs are mounted in a fashion similar to customer installations, e.g., using special racks that duplicate backplane mounting or box installation. Most packs are monitored continuously by software during PSEST, some with their own self-test. However, for some packs, power is switched off during temperature changes (ramps) and switched on when the temperature is constant (dwells).

When the product is new, the test setup is designed to provide a roughly equal temperature rise and fall for every pack so each receives approximately equal stresses. The setup remains the same for the entire life cycle of the product. To maintain a constant thermal mass in the chamber, a roughly homogeneous temperature among the packs, and maximum efficiency, the chamber is generally full during testing.

The circuit pack used as an example in this study is the control pack of an outdoor-installed asymmetric digital subscriber line (ADSL) product. The test in this case was an 8-hour (hr) test, with an initial $0.5-\mathrm{hr}(30-\mathrm{min})$ period at $25^{\circ} \mathrm{C}\left(77^{\circ} \mathrm{F}\right)$, followed by 10 cold $\left(-20^{\circ} \mathrm{C}\left[-4{ }^{\circ} \mathrm{F}\right]\right)$ and hot $\left(65^{\circ} \mathrm{C}\left[149^{\circ} \mathrm{F}\right]\right)$ dwells of $0.31 \mathrm{hr}(18.6 \mathrm{~min})$ duration. Figure 1 shows an outline of the temperature cycle. A similar analysis for an indoor-installed optical pack is described elsewhere. ${ }^{4}$ 


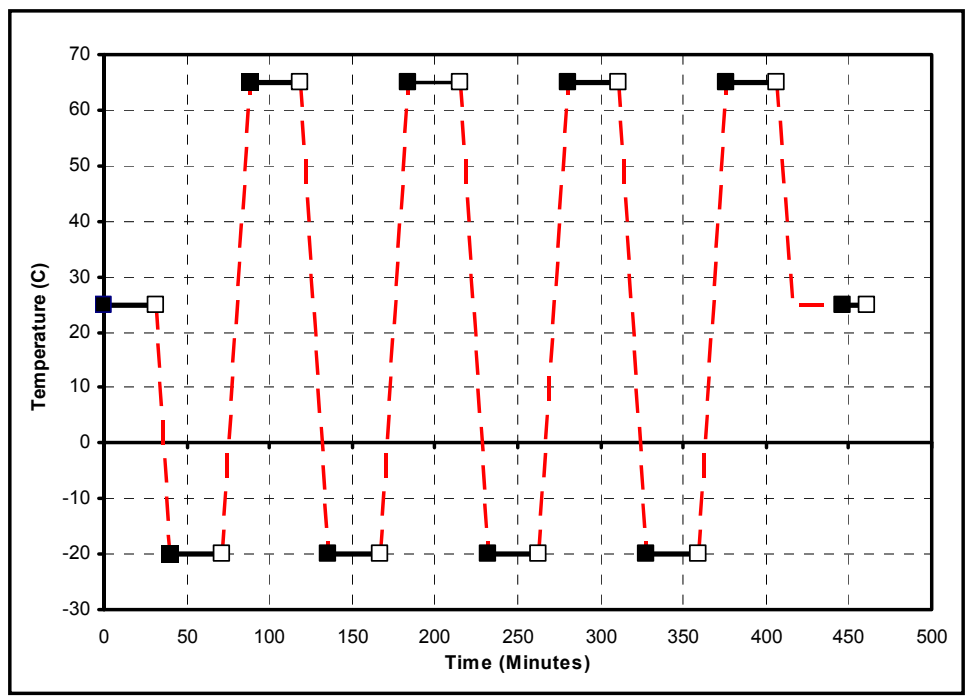

Figure 1. An outline of the temperature cycle of the example pack. Black squares and the full line indicate when the product is switched on; white squares and broken lines indicate when the product is turned off.

Repair technicians conduct troubleshooting and repairs in-house. The next level of hardware analysis is performed by engineers who monitor the repair data continuously and gather information that may contribute to product improvement. In this particular case, a component was sent for further analysis to the supplier and subsequently replaced by a similar, more reliable device. Early in the pack life cycle, design engineering may take samples of failing packs for further analysis.

It is important to emphasize that PSEST refers only to production testing, not laboratory testing, which is considered part of DEST. In the authors' experience, if PSEST and DEST have been effective, then the defects seen in customer service should be different from those seen in design and production. The authors have found that it is impossible to duplicate all the conditions of customer use, and thus strongly recommend incorporating investigation of field returns into any reliability program.

\section{THE RISK SET MODEL}

The key to understanding PSEST is the concept of the "risk set," which captures the dynamics of the test. This term did not originate here ${ }^{5}$ and has been used before in slightly different forms. ${ }^{6}$

The risk set consists of a subset of the population with weaknesses that have arisen from the manufacturing process. Examples are weak solder joints, poor assembly, and loose hardware. These weaknesses are in addition to defects that may have arisen from components or from design, to which all the units in the general population are susceptible. In a single short test, units with manufacturing weaknesses may be indistinguishable from the general population, but under environmental acceleration, the latent defects become actual failures and are detected by the test software.

It is possible for a member of the risk set to (apparently) rejoin the general population if the environmental acceleration is insufficient to precipitate it all the way to failure. This could happen if the test is ended prematurely. Sometimes such units are detected in a subsequent test, but they may also survive to reach the field. 
Note also that the risk set for PSEST contains only a subset of the general population affected by miscellaneous manufacturing-related defects. The general population also contains problems relating to design or components that are undetectable in PSEST, and may also reach the customer.

Hence, it is important to have both design and manufacturing controls, of which DEST and PSEST are key features.

\section{Development of the Model}

It is clear from the foregoing that the risk set should diminish over the duration of the test. Otherwise, it would be constant or increasing, thereby negating the concept of a limited risk set and suggesting an epidemic condition, generally beyond the scope of PSEST. It is true that PSEST sometimes detects epidemic conditions before they reach the field, but that is not its primary purpose. Other controls, such as adequate qualification of design and component changes, are recommended in preference.

For simplicity, it is assumed that only packs with a single defect type are included in the risk set. Then the failure function in PSEST is:

$$
F(t \mid P S E S T)=q F_{R S}(t \mid P S E S T)
$$

where $q$ is the proportion of the defect in the population $(0<q<1), F_{R S}($.$) is the cumulative failure$ function associated with the defect, and $t$ is a time variable.

An elaboration of this simple assumption is that the risk set is composed of packs with $M$ defect types. A simple model that corresponds to the risk set model discussed earlier is given by:

$$
F(t \mid P S E S T)=\sum_{i=1}^{M} q_{i} F_{R S i}(t \mid P S E S T)
$$

where $q_{i}$ is the proportion of the $i$ th defect type in the population $\left(0<q_{i}<1\right), F_{R S i}($.$) is the$ cumulative failure function of the $i$ th defect type, and $t$ is a time variable.

The assumption is that no pack fails with more than a single failure. This is reasonable when the average defects per pack is less than 0.1, i.e. the Poisson probability of two or more defects on any single pack is $<0.01$. The model can be fitted to each defect type, and the failure function of equation 2 constructed accordingly.

\section{Fitting a Parametric Model to Each Section}

The model chosen was a Weibull regression $\operatorname{model}^{7}$ with the regression equation:

$$
\begin{aligned}
\operatorname{Ln}\left(t_{p} \mid x, \beta\right) & =\beta_{0}+\underline{\beta .} \underline{x}^{\prime}+\sigma \Phi^{-1}(p) \\
& =\beta_{0}+\sum_{j=1}^{s} \beta_{j} x_{j}+\sigma \Phi^{-1}(p)
\end{aligned}
$$

where, $t_{p}$ is the $p$ th quantile, $\underline{x}$ is a vector of covariates $\left[x_{1} \ldots x_{s}\right]$, is a vector of coefficients estimated from the data $\left[\beta_{1} \ldots \beta_{s}\right], \beta_{0}$ is a parametric constant, $\sigma$ is a parameter of the Weibull distribution $\left(=1 / \alpha\right.$, where $\alpha$ is the shape parameter), and $\Phi^{-1}(p)$ is the $p$ quantile of the Weibull distribution $=\operatorname{Ln}[\operatorname{Ln}(1-p)]$. The regression coefficients $\left(\underline{\beta}, \beta_{0}, \sigma\right)$ are estimated from the data using the methods of Weibull regression. ${ }^{1}$ 
The PSEST data is broken into separate and sequential sections, with the survivors from the preceding section forming the units under test for the incoming section. Thus, for $K$ sections of testing (ramps, dwells, etc.), the data is treated as coming from $K$ distinct tests with a regression parameter that captures the age of the unit going into the test. The regression parameter captures the difference between the tests. It can be thought of as a growth parameter because at each successive test, the packs are more "resistant" to the stresses.

For each pack, information is extracted from the records database regarding test history. For each section of the test, a pack is recorded as having failed at a certain time in the section, or has a censoring time at the end of the section. For each section, the temperature and the time elapsed from the start of the section, called the "age" variable, are known.

For the $k$ th section, the times to failure are given by $t_{k}$ and the covariate values given in the $k$ th section by $x_{k}$ :

$$
\operatorname{Ln}\left(t_{k p} \mid x, \beta\right)=\beta_{0}+\sum_{j=1}^{s} \beta_{j} x_{k j}+\sigma \Phi^{-1}(p)
$$

After some experimentation, the covariates chosen were:

- Absolute temperature, suitably transformed to give figures in the region $[-10,10]$

- $\quad$ Ramp/dwell, a categorical variable $=0$ for a dwell and $=1$ for a ramp

- Age, being the time survived before entering the current section.

Using this structure, the data was submitted by the survreg() function of the package survival ${ }^{8}$ in the freeware statistical software $\mathrm{R},{ }^{9}$ which provided estimates of the coefficients $\underline{\beta}, \beta_{0}$, and $\sigma$.

\section{Example}

A typical pack has a PSEST of 8 hours duration, during which the pack experiences 10 temperature dwells at hot and cold temperatures. Testing is carried out only during the dwells, so no data is available on the ramps. Table 1 exhibits data from a sample of 717 packs.

Table 1. Production environmental test data for 717 packs.

\begin{tabular}{|l|l|l|l|r|l|}
\hline $\begin{array}{l}\text { Section } \\
\text { No. }(\boldsymbol{k})\end{array}$ & $\begin{array}{l}\boldsymbol{a}_{\boldsymbol{k}} \\
(\mathbf{h r s})\end{array}$ & \# of Packs & Failure Times in Section & Temp & $\boldsymbol{r}_{\boldsymbol{k}}$ \\
\hline 1 & 0.67 & 717 & 0.13 .0 .22 & $25^{\circ} \mathrm{C}$ & 0.9972 \\
\hline 2 & 1.47 & 715 & $\begin{array}{l}0.12,0.13,0.15,0.15,0.15,0.15,0.17,0.18, \\
0.2,0.22,0.22,0.22,0.23,0.23,0.3\end{array}$ & $-20^{\circ} \mathrm{C}$ & 0.9790 \\
\hline 3 & 2.28 & 700 & $0.13,0.15,0.17,0.27,0.28$ & $60{ }^{\circ} \mathrm{C}$ & 0.9929 \\
\hline 4 & 3.08 & 695 & $0.15,0.15,0.2,0.25,0.27$ & $-20{ }^{\circ} \mathrm{C}$ & 0.9928 \\
\hline 5 & 3.88 & 690 & & $60{ }^{\circ} \mathrm{C}$ & 1 \\
\hline 6 & 4.70 & 690 & $0.08,0.1,0.1$ & $-20{ }^{\circ} \mathrm{C}$ & 0.9927 \\
\hline 7 & 5.50 & 687 & 0.03 & $60{ }^{\circ} \mathrm{C}$ & 0.9985 \\
\hline 8 & 6.32 & 686 & $0.07,0.1,0.15$ & $-20^{\circ} \mathrm{C}$ & 0.9956 \\
\hline 9 & 7.12 & 683 & & $60{ }^{\circ} \mathrm{C}$ & 1 \\
\hline 10 & 7.93 & 683 & & $-20^{\circ} \mathrm{C}$ & 1 \\
\hline $\begin{array}{l}a_{k} \text { means the end of section } k ; \mathrm{r}_{k} \text { is the survival fraction in section } k \text {. These failures were classified as component } \\
\text { failures. }\end{array}$
\end{tabular}

For dwell $k$, the model is:

$$
\operatorname{Ln}\left(t_{p k}\right)=\beta_{0}+\beta_{1} \operatorname{Temp}_{k}+\beta_{2} a_{k-1}+\sigma \operatorname{Ln}[-\operatorname{Ln}(1-p)]
$$


Temp is the temperature variable, suitably transformed:

$$
\operatorname{Temp}_{k}=10^{8}\left\{\frac{1}{273.16+f}-\frac{1}{273.16+c_{k}}\right\}
$$

where, $f$ is the temperature outside the chamber (taken to be $25^{\circ} \mathrm{C}\left[77^{\circ} \mathrm{F}\right]$ ); $c_{k}$ is the temperature inside (in ${ }^{\circ} \mathrm{C}$ ) during section $k ; a_{k-1}$ is the endpoint of the previous dwell, or the "age" of the pack entering section $k$; and $t_{p k}$ is the $p$ th quantile in section $k$ measured from the start of the section.

Data is presented in a form suitable for the survreg function in the R package survival. Each pack is coded as a distinct entry with the covariate values in each dwell, using a censoring code of 1 for a failure and 0 for a passing pack.

The coefficient values for this model are estimated as:

\begin{tabular}{|l|l|}
\hline Coefficient & Estimate \\
\hline$\beta_{0}$ & 9.9535 \\
\hline$\beta_{1}$ & -0.0844 \\
\hline$\beta_{2}$ & 0.7303 \\
\hline$\sigma$ & 1.570 \\
\hline
\end{tabular}

Note that the coefficient of Temp is negative as it tends to reduce time to failure, while the coefficient of "age" is positive as it tends to lengthen time to failure.

The Weibull shape parameter, $\alpha=1 / \sigma$, in this case equals 0.6369 . The plots of the actual percentage of packs surviving and the fitted reliability function are given in Figure 2.

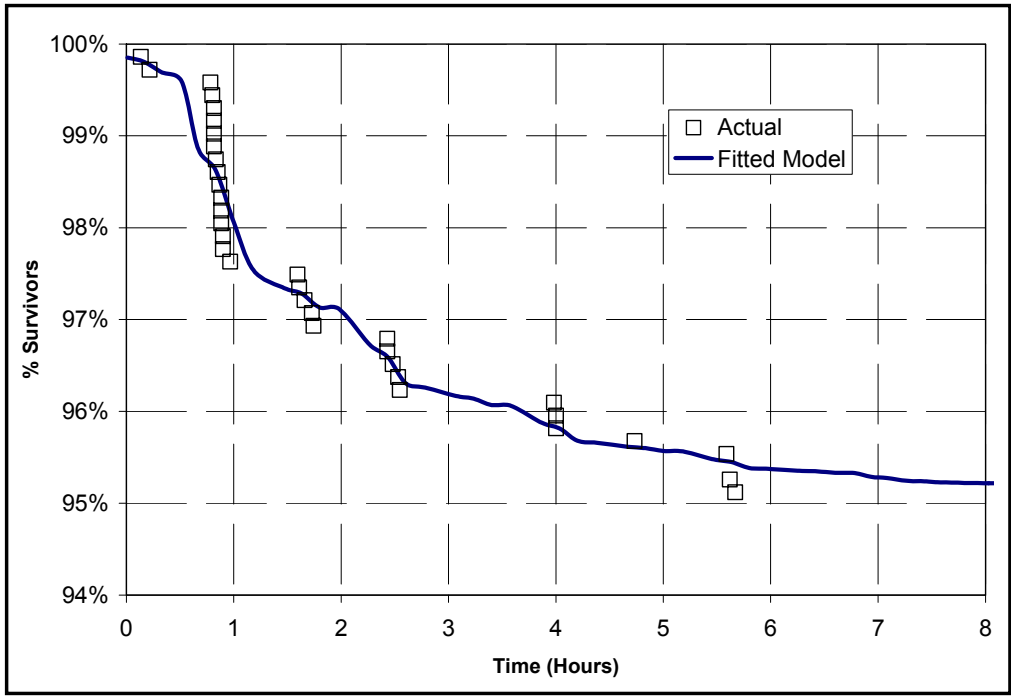

Figure 2. Reliability function of PSEST data and fitted model. The squares give the plot of the empirical failure function, plotted from the data. The full line is a plot of the fitted reliability function. 


\section{Simplification of the Model}

A simpler model investigated was based on Poisson regression from the class of generalized linear models. ${ }^{10}$ In this model, the natural $\log$ of the failure count is linearly dependent on the covariates. In test section $k$,

$$
\log \left(N_{k}\right)=\beta_{g 0}+\beta_{g 1} \operatorname{Temp}_{k}+\beta_{g 2} a_{k-1}+\log \left(S_{k-1} D_{k}\right)
$$

where $N_{k}$ is the number of failures in section $k, S_{k-1}$ is the number of survivors from section $k-1$, and $D_{k}$ is the length of section $k$. The other variables are defined as in equation 5 .

Using the $\operatorname{glm}()$ function in $\mathrm{R}$, the following estimates were obtained:

\begin{tabular}{|l|l|}
\hline Coefficient & Estimate \\
\hline$\beta \mathrm{g}_{0}$ & -5.0982 \\
\hline$\beta \mathrm{g}_{1}$ & 0.0590 \\
\hline$\beta \mathrm{g}_{2}$ & -0.4328 \\
\hline
\end{tabular}

This model is easier to handle, as it requires only the results of each individual section of the test. Also, it is possible to show with some algebra that the models given by equations 5 and 7 are identical when $1-r_{k}$ is small, and $\beta_{p i}=-\beta_{w i} \alpha$.

A further development of this model is apparent by putting $N_{k} / S_{k-1}=1-r_{k} \approx-\log \left(r_{k}\right), N_{k}>0$. Equation 7 can be written in the form:

$$
\log \left[-\log \left(r_{k}\right)\right]=\log \left(D_{k}\right)-\beta_{g 2} D_{k}+\beta_{g 0}+\beta_{g 1} \operatorname{Temp}+\beta_{g 2} a_{k}
$$

This is a linear equation in the form $y=b_{0}+b_{1} x_{1}+b_{2} x_{2}$ with covariates temperature and time.

Using the data from Table 1, the following estimates were obtained from an ordinary least squares fit:

\begin{tabular}{|l|l|}
\hline Coefficient & Estimate \\
\hline$\beta \mathrm{g}_{0}$ & -5.3462 \\
\hline$\beta \mathrm{g}_{1}$ & 0.0476 \\
\hline$\beta \mathrm{g}_{2}$ & -0.3122 \\
\hline
\end{tabular}

These figures are close to the estimates obtained above, though the fit of the model is not as $\operatorname{good}\left(R^{2}=0.8342\right)$. However, this linear model is quite useful for exploratory analysis.

Further, equation 8 can be adapted:

$$
r_{k}=\exp \left[-\exp \left(\frac{a_{k}-\eta_{k}}{\delta}\right)\right]
$$

where $\delta=1 / \beta_{g 2}, \eta=-C-\beta_{g 1} \boldsymbol{T} \boldsymbol{e m p} \boldsymbol{p}_{\mathrm{k}}$, and $C=\log \left(D_{k}\right)-\beta_{g 2} D_{k}+\beta_{g 0}$ from equation 8 .

This is the cumulative distribution function of an Extreme Value (or Gumbel) distribution. Therefore, as $k \rightarrow \infty, r_{\infty} \rightarrow 1$, which implies that eventually the units that have survived all preceding sections of the test will survive with probability 1 in the test. This is the risk set model. 
Equation 9 can be used to estimate $q$ because the reliability at the end of section $k$ is the multiple of the preceding reliabilities:

$$
F(t \mid P S E S T)=q F_{R S}(t \mid P S E S T)=1-\prod_{i=1}^{k} r_{i}
$$

where $t=a_{k}$.

Therefore, using the estimates of $r_{k}$, the estimate of $q$ is:

$$
\widehat{q}=1-\operatorname{Lim}_{k \rightarrow \infty} \prod_{i=1}^{k} \hat{r}_{k}
$$

In the example used, the estimate is $\hat{q} \approx 5 \%$. The limit for $R($.$) can be estimated graphically$ from Figure 2 . Using the estimate $\hat{q} \approx 5 \%$, the actual $\%$ failure is $33 / 717=0.46 \%$, giving an estimate of escapes as $0.4 \%$.

\section{A SIMPLE COST MODEL}

If $\tau$ is the end of test, then the fraction of risk set escapes from test at time $t>\tau$ is given by $\Delta E(t)=q F_{R S}(t \mid t>\tau)-q F_{R S}(\tau \mid P S E S T)$. If $w$ is the end of the warranty period, then we can assume that $F_{R S}(w)=1$ so $\Delta E(w)=q\left[1-F_{R S}(\tau \mid P S E S T)\right]$.

Let the non-risk set failures by given by $F_{N R S}(t), t>0$, where $F_{N R S}(\tau)=0$. Since a nonrisk set defect can occur on any pack, the failure function in the field is:

$$
F(t \mid \text { Field })=\left[1-F_{\text {NRS }}(t)\right] \Delta E(t)+F_{N R S}(t), t>\tau
$$

In particular, at the end of warranty:

$$
F(w \mid \text { Field })=\left[1-F_{\text {NRS }}(w)\right] \Delta E(w)+F_{\text {NRS }}(w), w>\tau
$$

Then, a model for the total cost of failure in test and warranty is given by:

$$
T C(\tau)=A I(\tau)+B \tau+C q F(\tau \mid P S E S T)+D F(w \mid \text { Field })
$$

where, $A=$ fixed cost of test, $B=$ variable cost of test per hour, $C=$ cost of an internal repair, $D=$ cost of a warranty repair, and $I(\tau)=0$ when $\tau=0, I(\tau)=1$ otherwise. Equation 14 can be minimized to give an optimum length of PSEST. In this case, a duration of 6 hours for PSEST was shown to be optimal.

\section{CONCLUSIONS}

The models described here essentially model the transient period at the start of the lifetime of a product, when it is subject to weaknesses that have arisen from the manufacturing process. Problems that might arise in the longer term from intrinsic design, component weaknesses, or extrinsic influences such as corrosion are best addressed by testing at the design stage. 
Therefore, for longer-term reliability tests, suitable acceleration models such as the CoffinManson model for the number of cycles to failure are better employed. ${ }^{11}$ However, some initial studies have shown that the models described here are also applicable after the onset of wearout.

The models described in this paper have been applied across a large sample of pack types. The results of this work have been of significant benefit for the management of PSEST and have formed the basis for a rationalization of the process. Analytical modeling and cost modeling was included in all process and product reviews to ensure efficient testing. PSEST is a significant process bottleneck, and the model ensures its continuing efficiency and effectiveness. The test can be shortened progressively as the product matures and yield improves, as is the norm.

Significant savings arise from faster throughput times, reduction in operator support, shorter queues, and lower in-process inventory. Other savings result from lower power usage and maintenance costs.

From the definition of test escapes, $\Delta E(t)$, it is clear that testing cannot be reduced without a small percentage increase in the shipment of packs that will fail prematurely in the field. This increase is kept as small as possible and is usually offset by the gradual product improvement that comes from PSEST.

In 2006-07, savings of approximately $\$ 5$ million were estimated from optimized PSEST, with minimal impact on field reliability. In addition, much has been learned on the topic of production environmental testing:

\section{Key Learning}

(1) PSEST is a natural follow-on from a DEST/HALT program.

(2) PSEST can provide cost-effective detection and repair of early life failures, which would otherwise occur at the customer site.

(3) Despite often being compared unfavorably to HASS, PSEST (with lower-level stresses than HASS) is worth retaining as a manufacturing screen or sample check, provided it is accompanied by effective management and analysis.

(4) PSEST can adapt to changing circumstances; that is, duration can be shortened, sampling can be introduced, or the test can be eliminated.

(5) The most successful PSEST operations use temperature cycling. Optimization is achieved by tailoring the number of temperature dwells to obtain a cost-effective test.

(6) Management of PSEST is most effective with full cooperation of managers, test engineers and reliability engineers.

\section{Recommendations for a Successful PSEST Program}

(1) Temperature cycling with a temperature "delta" of at least $50{ }^{\circ} \mathrm{C}\left(122{ }^{\circ} \mathrm{F}\right)$ is recommended. This removes any distinction between types of temperature cycling. The stresses should be the maximum possible under the circumstances and should not be based on expected field conditions.

(2) Make the temperature delta as large as the equipment can provide. It is acceptable to go outside the pack specification limits. In making these decisions, be guided by the DEST/HALT experience or consult with someone who has experience with DEST and HALT.

(3) Maximize the number of cycles in the time available, that is, use the highest rate of change the equipment can provide. If the rate of change is low, carry out at least two full cycles.

(4) Allow sufficient dwell time for all the packs (at full chamber load) to reach the maximum and minimum temperature, plus time to test all of the packs.

(5) Check that the chamber is characterized at full load, using a chart recorder.

(6) Use a good data gathering system.

(7) The software must capture the time to failure. This is essential for any assessment of PSEST effectiveness. The models used here demand at least the number of dwells (hot and cold) before failure. 
(8) Record and store data on packs that are removed for repair.

(9) Knowing the types of defects captured is essential for a good program.

- Analyze defects and compare with field failures. The risk set model implies that if the percentage fallout in PSEST is close to the estimated $q$, then the problem types seen in PSEST should not be repeated in the field.

- Start modeling when the process is stable, that is, when stable, appreciable numbers of packs are operating in the field without major problems. This may take up to a year to achieve.

(10) Repeat the analysis periodically and act according to its results.

\section{ACKNOWLEDGMENTS}

The research for this paper was supported by IDA-Ireland. The authors gratefully acknowledge good advice from the reviewers of this paper, and assistance from Dr. John Donovan and Bahman Honari of the Centre for Telecommunications Value-Chain Research (CTVR), Institute of Technology Sligo, Sligo, Republic of Ireland. Contributions from outside Alcatel-Lucent were supported by Science Foundation Ireland (SFI) under SFI grant 03/CE3/1405.

\section{REFERENCES}

1. Meeker, W.Q. and L. Escobar. 1998. Statistical Models for Reliability Data. New York: Wiley.

2. McCullagh, P. and J. A. Nelder. 1999. Generalized Linear Models, 2nd ed. Boca Raton: Chapman and Hall/CRC.

3. Hobbs, G. 2000. Accelerated Reliability Engineering: HALT and HASS. New York: Wiley.

4. Joyce, T., E. Lisay, D. E. Dalton, J. M. Punch, M. S. Shellmer, S. N. Kher, and S. Goyal. 2006. Piecewise Modeling of Circuit Pack Temperature Cycling Data. Bell Labs Technical Journal, 11 (3): 21-37.

5. Honari, B., J. Donovan, T. Joyce, and S. Wilson. 2008. Application of Generalized Linear Models for Optimizing Production Stress Testing. Proceedings of the Annual Reliability and Maintainability Symposium.

6. Honari, B., J. Donovan, and S. Wilson. 2007. Use of an Integrated Production Test and Field Reliability Model for Process Optimization. MMR Mathematical Methods in Reliability: Methodology and Practice Conference Proceedings.

7. Ruppert, D., M. P. Wand, and R. J. Carroll. 2003. Semiparametric Regression. Cambridge, UK: Cambridge University Press.

8. Jensen, F. and N. E. Petersen. 1982. Burn-In: An Engineering Approach. New York: Wiley.

9. Therneau, T. M. and T. Lumley. Survival: Survival Analysis using Penalized Likelihood. R package, http://cran.r-project.org/web/packages/survival/index.html.

10. R Development Core Team. 2004. R: A Language and Environment for Statistical Computing. useR! (International R User) Conference. http://www.R-project.org.

11. Meeker, W. Q. and L. Escobar. 2006. A Review of Accelerated Test Models. Statistical Science 21:552-577.

\section{ABOUT THE AUTHORS}

Toby Joyce is a member of the technical staff and statistical analyst in the ALU Reliability Physics group. He has an MS in mathematical science from the National University of Ireland, Galway, Ireland, and an MS in statistics from Trinity College, Dublin, Ireland. His main professional interests are field data analysis, reliability improvement, and the application of statistics to manufacturing and logistics. 
Oonagh Gaffney is senior test engineer for Alcatel-Lucent Digital Subscriber Line (DSL) products managed from Blanchardstown, Ireland. Gaffney received a BS from the Open University (UK) and a Masters in engineering from the Dublin Institute of Technology (2007). Her main professional interest is in improved IT tools for remote pack testing and information sharing across the Internet.

Simon Wilson is professor of statistics at Trinity College, Dublin. Wilson received a $\mathrm{PhD}$ in Stochastic Modeling from George Washington University in 1993. His research interests are the use of Bayesian methods in reliability and other fields of science and engineering.

Shirish Kher is technical manager of the Optics Reliability Group and is responsible for reliability of optical products at Alcatel-Lucent. He has a $\mathrm{PhD}$ in engineering mechanics from Pennsylvania State University. He has received multiple Bell Labs Presidents' Awards. His interests and experience in the field range from reliability physics to network architecture and analysis.

Contact author: Toby Joyce, Reliability Physics, Alcatel-Lucent, Blanchardstown Industrial Estate, Snugboro Rd., Dublin 15, Ireland; e-mail: tobyjoyce@alcatellucent.com. 